\title{
Extension of Hardy's class for Ramanujan's interpolation formula and master theorem with applications
}

\author{
Muhammed Aslam Chaudhry ${ }^{{ }^{*}}$ and Asghar Qadir $^{2}$
}

\footnotetext{
* Correspondence: maslam@kfupm. edu.sa

${ }^{1}$ Department of Mathematics and Statistics, King Fahd University of Petroleum and Minerals, Dhahran, 31261, Saudi Arabia

Full list of author information is available at the end of the article
}

\author{
Abstract \\ Hardy's class of functions for the Ramanujan interpolation formula and Ramanujan- \\ Hardy master theorem is extended to a wider domain of applicability and the \\ extension is illustrated by examples. \\ MSC: 11M06, 11M26, 11M36, 11M99, 42 A32, 11M06, 42A32
}

Keywords: Gamma function, zeta function, Hurwitz's zeta function, Mellin transforms, Weyl transform, Hardy-Ramanujan's master theorem

\section{Introduction}

Ramanujan was in a class by himself as an intuitive mathematician and provided many results that have kept generations of mathematicians busy to prove them rigorously (see $[1,2])$. Given the accuracy of his intuition it may be wondered why it is worth the effort of proving the remaining results rigorously. Of course, it is worth while as the next intuition may prove wrong (see [[2], p. 188(11.3)]). More importantly, the domain of applicability of the correct results needs to be specified. A case in point is the interpolation formula and the master theorem arrived at intuitively by Ramanujan and proved by Hardy, now known as the Hardy-Ramanujan master theorem [[3], p. 189]. For the interpolation formula, which is used in proving the master theorem, Hardy proved it using the domain $(0, \pi)$ for the parameter $A$ that appeared in the proof. He remarked that it may be true for the value $A=\pi$ as well but that he 'did not have time for any more subtle argument' (see [[2], p. 191]), seeming to imply that this extension was unimportant and/or too complicated. The proof does not seem to have appeared anywhere else in the literature since. However, it turns out that this constraint seriously limits the domain of applicability of the theorem as most of the commonly used functions do require $A=\pi$. In this article, we prove the result rigorously for $A \in(0, \pi]$ and then extend the theorem beyond its original context. More precisely, Hardy considered the space of analytic functions [[2], p. 189] as follows. Take $0<\delta<1$ and $H(\delta):=\{s=\sigma+i \tau: \sigma \geq-\delta\}$ as the half space. Suppose that $0<\mathrm{A}<\pi$ and

$$
\Re=\Re(A, P, \delta):=\left\{\phi(s\}:|\phi(s)| \leq C e^{P \sigma+A|\tau|}, s \in H(\delta)\right\} .
$$

The space $\mathfrak{R}$ is called the Hardy space of analytic functions that restricts the parameter $A$ to lie in $(0, \pi)$. Consider a function $\varphi \in \Re$ and define [[2], p. 189]

(c) 2012 Chaudhry and Qadir; licensee Springer. This is an Open Access article distributed under the terms of the Creative Commons Attribution License (http://creativecommons.org/licenses/by/2.0), which permits unrestricted use, distribution, and reproduction in any medium, provided the original work is properly cited. 


$$
\Phi(x):=\frac{1}{2 \pi i} \int_{c-i \infty}^{c+i \infty} \frac{\pi}{\sin \pi s} \phi(-s) x^{-s} \quad(0<c<\delta) .
$$

The integrand in (1.2) is majorized, for all positive $x$, by a constant multiple of

$$
\mathrm{e}^{-(\pi-A)|\tau|} \mathrm{e}^{-P c} x^{-c},
$$

and the integral (1.2) converges uniformly in an interval $0<x \leq x_{0} \leq X<\infty$. Therefore, the function $\Phi(x)$ is regular, and represented by the integral (1.2), for all positive $x$.

For completeness we state the 'canonical' form of the theorem, the Hardy-Ramanujan master theorem: If in some neighborhood of $x=0$,

$$
\Phi(x)=\sum_{n=0}^{\infty} \frac{(-1)^{n} \phi(n) x^{n}}{n !}
$$

for some analytic function $\varphi(s)(\sigma>-\delta)$, then

$$
\int_{0}^{\infty} \Phi(x) x^{s-1} \mathrm{~d} x=\int_{0}^{\infty}\left(\sum_{n=0}^{\infty} \frac{(-1)^{n} \phi(n) x^{n}}{n !}\right) x^{s-1} \mathrm{~d} x=\Gamma(n) \phi(-n) .
$$

By Cauchy's theorem (see $[2,4]$ for example) for (1.2), we obtain the series representation

$$
\Phi(x)=\frac{1}{2 \pi i} \int_{c-i \infty}^{c+i \infty} \frac{\pi}{\sin \pi s} \phi(-s) x^{-s}=\sum_{n=0}^{\infty}(-1)^{n} \phi(n) x^{n} \quad\left(\phi \in \Re, 0<x<\mathrm{e}^{-P}\right)
$$

which is another form of the Hardy-Ramanujan's master theorem. This leads to the Ramanujan interpolation formula

$$
\int_{0}^{\infty} x^{s-1}\left\{\phi(0)-\phi(1) x+\phi(2) x^{2}-\ldots\right\} \mathrm{d} x=\frac{\pi}{\sin \pi s} \phi(-s) .
$$

Since, for $\varphi(x)=\sin \pi x$ the left hand side (LHS) in (1.7) is zero while the right hand side is $-\pi$, it is clear, as indicated by Hardy (see [[2], p. 188]), that the formula (1.7) is not applicable as such, there must be a constraint on $\varphi(s)$. The Hardy class $\mathfrak{R}=\mathfrak{R}(A$, $P, \delta$ ) does not accommodate functions of common interest. We give applications of the master theorem by introducing a larger class of functions using Mellin and Weyl transforms. It may be noted that the Riemann zeta function

$$
\zeta(s):=\sum_{n=1}^{\infty} \frac{1}{n^{s}} \quad(s:=\sigma+i \tau, \sigma>1),
$$

was originally introduced by Euler (for real $s$ ) who proved that $[[5]$, p. 1]

$$
\zeta(s)=\prod_{p}\left(1-p^{-s}\right)^{-1} \text { (where } p \text { runs through all primes) }
$$

which plays an important role in the study of prime numbers. Riemann proved that the zeta-function has a meromorphic continuation to the complex plane and satisfies the Riemann functional equation (see [[5], p. 13 (2.1.1)]) 


$$
\zeta(s)=2(2 \pi)^{-(1-s)} \cos \left(\frac{\pi}{2}(1-s)\right) \Gamma(1-s) \zeta(1-s) .
$$

The zeta function has simple zeros at $s=-2,-4,-6, \ldots$, called the trivial zeros. All the other zeros, called the non-trivial zeros, of the function are symmetric about the critical line $\sigma=1 / 2$, in the critical strip $0 \leq \sigma \leq 1$. The multiplicity of these non-trivial zeros (in general) is not known. Riemann conjectured that the non-trivial zeros of the function lie on the critical line $\sigma=1 / 2$. This conjecture is called the Riemann hypothesis. Define

$$
\chi(s):=2(2 \pi)^{-(1-s)} \cos \left(\frac{\pi}{2}(1-s)\right) \Gamma(1-s) .
$$

Then the functional equation (1.11) can be rewritten as

$$
\zeta(s)=\chi(s) \zeta(1-s) .
$$

Using the identity

$$
\frac{\pi}{\sin \pi s}=\Gamma(s) \Gamma(1-s),
$$

for the gamma function, we can rewrite the function $\chi(s)$ as

$$
\chi(s)=\frac{\pi^{s-\frac{1}{2}} \Gamma\left(\frac{1}{2}-\frac{1}{2} s\right)}{\Gamma\left(\frac{1}{2} s\right)} .
$$

For basic definitions, historical background and results involving gamma and zeta functions, we refer to the standard references [1-3,5-12]. We have shown applications of the general lemma by considering the functions that involve the gamma, the Riemannzeta and the $\chi(s)$ functions.

The plan of the article is as follows: we briefly review the aspects of the Mellin and Weyl transforms that we need to use in the next section. We introduce the notation for the Weyl transform of a function that is found helpful in stating the master theorem and other related results precisely. We prove a general lemma in Section 3 that shows the existence of the inverse Mellin transform (IMT) of a class of functions accommodating the case $A=\pi$. Some applications of the general result are also shown. We define three classes of functions in Section 4 that are suitable for HardyRamanujan's master theorem for different intervals of the variable $x$ appearing in the series representation of the IMT. Some special cases of the theorem are discussed in Section 5. Different forms of Ramanujan's master theorem are shown to follow from the general lemma in the Section 6. Some concluding remarks are given in the final section.

\section{Preliminaries}

The function spaces $\mathrm{H}(\kappa ; \lambda)$ and $\mathrm{H}(\infty ; \lambda)$ are defined as follows: A function $f \in C^{\infty}(0, \infty)$ is said to be a member of $\mathrm{H}(\kappa ; \lambda)$ if

1. $f(t)$ is integrable on every finite subinterval $[0, T](0<T<\infty)$ of $\mathbb{R}_{0}^{+}:=[0, \infty)$,

2. $f(t)=O\left(t^{-\lambda}\right)\left(t \rightarrow 0^{+}\right)$, 
3. $f(t)=O\left(t^{-\kappa}\right)(t \rightarrow \infty)$.

Furthermore, if the above relation $f(t)=O\left(t^{-\kappa}\right)(t \rightarrow \infty)$ is satisfied for every exponent $\kappa \in \mathbb{R}_{0}^{+}$, then the function $f(t)$ is said to be in the class $\mathrm{H}(\infty ; \lambda)$. It is to be noted that $\mathrm{H}(\infty ; \lambda) \subset \mathrm{H}(\kappa ; \lambda)\left(\forall \kappa \in \mathbb{R}_{0}^{+}\right)$. Clearly, we have

$$
f(t)=e^{-b t} \in \mathrm{H}(\infty, 0) \quad(b>0) .
$$

The Mellin transform of $f \in \mathrm{H}(\kappa ; \lambda)$ is defined by [[13], p. 83],

$$
f_{M}(s):=M[f(t) ; s]:=\int_{0}^{\infty} f(t) t^{s-1} \mathrm{~d} t \quad(s=\sigma+i \tau, \lambda<\sigma<\kappa) .
$$

The Weyl transform (or Weyl's fractional integral) of order $s$ of $\omega \in \mathrm{H}(\kappa ; \lambda)$ is defined by (see [[3], Vol. II, p. 181] and [[14], p. 237]),

$$
\begin{aligned}
& \Omega(s ; x):=W^{-s}[\omega(t)](x):=\frac{1}{\Gamma(s)} M[\omega(t+x) ; s] \\
= & \frac{1}{\Gamma(s)} \int_{0}^{\infty} \omega(t+x) t^{s-1} \mathrm{~d} t=\frac{1}{\Gamma(s)} \int_{x}^{\infty} \omega(t)(t-x)^{s-1} \mathrm{~d} t \quad(s=\sigma+i \tau, 0<\sigma<\kappa, x \geq 0) .
\end{aligned}
$$

For $\sigma \leq 0$, we define the Weyl transform (or Weyl's fractional derivative) of order $s$ of $\omega \in \mathrm{H}(\kappa ; 0)$ as follows (see [[14], p. 241]):

$$
\Omega(s ; x):=W^{-s}[\omega(t)](x):=(-1)^{n} \frac{d^{n}}{\mathrm{~d} x^{n}}(\Omega(n+s ; x)), \quad(0 \leq n+\sigma<k),
$$

where $n$ is the smallest positive integer greater than or equal to $-\sigma$ provided that $\omega(0)$ is well defined and that

$$
\Omega(0 ; x):=\omega(x) .
$$

We can rewrite Weyl's fractional derivative (2.4) alternately as

$$
\begin{aligned}
& \Omega(-s ; x):=W^{s}[\omega(t)](x)=(-1)^{n} \frac{d^{n}}{\mathrm{~d} x^{n}}\left(W^{-(n-s)}[\omega(t)](x)\right) \\
& =:(-1)^{n} \frac{d^{n}}{\mathrm{~d} x^{n}}(\Omega(n-s ; x))
\end{aligned}
$$

where $n$ is the smallest positive integer greater than or equal to $\sigma$. In particular for $s=n(n=0,1,2,3, \ldots)$ in $(2.6)$, we obtain

$$
\Omega(-n ; x):=W^{n}[\omega(t)](x):=(-1)^{n} \frac{d^{n}}{\mathrm{~d} x^{n}}(\Omega(0 ; x))=(-1)^{n} \frac{d^{n}}{\mathrm{~d} x^{n}}(\omega(x)) .
$$

Notice that $\left\{W^{s}\right\}(s \in \mathbb{C})$ is a multiplicative group [[14], p. 245] and satisfies

$$
W^{-(\mu+s)}[\omega(t)](x)=W^{-\mu}[\Omega(s ; t)](x)=\Omega(s+\mu ; x] .
$$

Following the above terminology, we obtain

$$
\Omega(0 ; x)=\sum_{n=0}^{\infty} \frac{(-1)^{n} \Omega(-n ; 0) x^{n}}{n !} \quad(\omega \in \mathrm{H}(\kappa ; 0), 0 \leq \sigma<k, x \geq 0) .
$$

Weyl transform notation is helpful for obtaining a precise formulation of the HardyRamanujan master theorem and Ramanujan's interpolation formula. 


\section{An extended form of the Hardy class for Ramanujan's master theorem}

Let $b \in(0,1]$ and $F(s)(\sigma<b)$ be an analytic function which is not identically zero at all the non-positive integers, $s=0,-1,-2,-3, \ldots$, and

$$
|F(s) \Gamma(s)|=O\left(|\tau|^{\varepsilon(\sigma-b)}\right) \quad\left(|\tau| \rightarrow \infty,-\infty<\sigma_{1} \leq \sigma \leq \sigma_{2}<b, \varepsilon \geq \varepsilon_{0}>0\right)
$$

Define

$$
\begin{aligned}
& f_{M}(s):=\Gamma(s) F(s) \quad(\sigma<b), \\
& f(z):=\frac{1}{2 \pi i} \int_{C} f_{M}(s) z^{-s} \mathrm{~d} s \quad(z \neq 0),
\end{aligned}
$$

where $C$ denotes a loop in the complex $s$ plane that encircles the poles $s=0,-1,-2$, $-3, \ldots$ of $\Gamma(s)$ (in the positive sense) that passes through $0<\sigma<b$ with end points at infinity in $\sigma<0$. We refer to [[13], p. 90] for the representation of the loop. If the loop $C$ approaches $\infty$ in the direction $\pm \theta_{0}$ say, where $\frac{1}{2} \pi<\theta_{0} \leq \pi$, then the logarithm of the modulus of the integrand on $C$ (where $s=\mathrm{Re}^{i \theta}$ ) in (3.3) behaves like $\varepsilon \mathrm{R} \cos \theta_{0} \log (\mathrm{R})+$ $O(R) \rightarrow-\infty$ as $R \rightarrow \infty$. Consequently, (3.3) defines $f(z)$ without any restriction on $\arg (z)$ and thereby represents an analytic continuation of the line integral

$$
f(z):=\frac{1}{2 \pi i} \int_{c-i \infty}^{c+i \infty} f_{M}(s) z^{-s} \mathrm{~d} s \quad\left(|\arg (z)|<\frac{1}{2} \pi, z \neq 0,0<c_{1} \leq c \leq c_{2}<b\right) .
$$

Note that the loop $C$ in (3.3) is bent back into the vertical path $\sigma=\operatorname{Re}(s)=c\left(0<c_{1}\right.$ $\left.\leq c \leq c_{2}<b\right)$, (3.3) is valid for $|\arg z|<\frac{1}{2} \pi$ and hence the integral is convergent only when $z=x>0$ and $0<c_{1} \leq c \leq c_{2}<b$. Therefore, the line integral

$$
f(x):=\frac{1}{2 \pi i} \int_{c-i \infty}^{c+i \infty} f_{M}(s) x^{-s} \mathrm{~d} s \quad\left(x>0,0<c_{1} \leq c \leq c_{2}<b\right),
$$

is well defined. Hence, we have proved the following lemma.

Lemma (3.1) Let $b \in(0,1]$ and $F(s)(\sigma<b)$ be an analytic function not identically zero at all the non-positive integers at $s=0,-1,-2,-3, \ldots$, and

$$
|F(s) \Gamma(s)|=O\left(|\tau|^{\varepsilon(\sigma-b)}\right) \quad\left(|\tau| \rightarrow \infty,-\infty<\sigma_{1} \leq \sigma \leq \sigma_{2}<b, \varepsilon \geq \varepsilon_{0}>0\right) .
$$

Then the inverse Mellin transform (3.5) is well defined and it can be evaluated by using Cauchy's residue theorem [[4], p. 84].

Remark (3.1) In the above lemma we note that $f_{M}(s):=\Gamma(s) F(s)(0<\sigma<b)$ is the well defined Mellin transform which is analytic in the strip. Therefore, following the notation of Section 2 we note that

$$
f_{M}(s):=\Gamma(s) F(s)=\Gamma(s) F(s ; 0) \quad(0<\sigma<b),
$$

where,

$$
\int_{0}^{\infty} F(0 ; x) x^{s-1} \mathrm{~d} x=\Gamma(s) F(s ; 0) \quad(0<\sigma<b) .
$$


Taking the inverse Mellin transform of both sides in (3.8), we find

$$
F(0 ; x):=\frac{1}{2 \pi i} \int_{c-i \infty}^{c+i \infty} \Gamma(s) F(s ; 0) x^{-s} \mathrm{~d} s \quad(0<c<b) .
$$

Since $F(s ; 0)(\sigma<b)$ is analytic function which is not identically zero at all the nonpositive integers at $s=0,-1,-2,-3, \ldots$, and

$$
|\Gamma(s) F(s ; 0)|=O\left(|\tau|^{\varepsilon(\sigma-b)}\right) \quad\left(|\tau| \rightarrow \infty,-\infty<\sigma_{1} \leq \sigma \leq \sigma_{2}<b, \varepsilon \geq \varepsilon_{0}>0\right),
$$

therefore, according to the above lemma, the inverse Mellin transform (3.9) is well defined. Application of Cauchy's theorem to (3.9) gives that

$$
F(0 ; x)=\sum_{n=0}^{\infty} \frac{(-1)^{n} F(-n ; 0) x^{n}}{n !} \quad(0 \leq x<\infty) .
$$

Equations (3.8) and (3.11) yield

$$
\int_{0}^{\infty} x^{s-1}\left(\sum_{n=0}^{\infty} \frac{(-1)^{n} F(-n ; 0) x^{n}}{n !}\right) \mathrm{d} x=\Gamma(s) F(s ; 0) \quad(0<\sigma<b),
$$

which is the Hardy-Ramanujan master theorem.

It is worth remarking that (3.12) can be extended further as

$$
F(s ; x)=\sum_{n=0}^{\infty} \frac{(-1)^{n} F(s-n ; 0) x^{n}}{n !} \quad(0 \leq x<\infty, 0 \leq \sigma<b),
$$

provided $F(s ; x)(\sigma<b)$ is analytic for all $x \geq 0$ and

$$
|\Gamma(s) F(s ; x)|=O\left(|\tau|^{\varepsilon(\sigma-b)}\right) \quad\left(|\tau| \rightarrow \infty,-\infty<\sigma_{1} \leq \sigma \leq \sigma_{2}<b, \varepsilon \geq \varepsilon_{0}>0, x \geq 0\right) .
$$

The asymptotic restriction (3.10) can be modified by allowing the series (3.11) to converge only in a finite interval $0<x<e^{-p}$ (see [[2], p. 189]), which is generally the case when the Riemann zeta function is involved. However, then the representation (3.12) is meaningless as the series will be divergent on the infinite interval $0<x<\infty$. To be precise, three type of asymptotic relations where the IMT (3.9) is well defined and the corresponding series converges in different intervals of the variable $x$ are needed.

Suppose that $F(s ; 0)(\sigma<b)$ is analytic function which is not identically zero at all the non-positive integers at $s=0,-1,-2,-3, \ldots$, and

$$
\begin{aligned}
& |\Gamma(s) F(s ; 0)| \leq C B^{\sigma} e^{-A|\tau|} \\
& \left(|\tau| \rightarrow \infty,-\infty<\sigma_{1} \leq \sigma \leq \sigma_{2}<b\right),
\end{aligned}
$$

where $A, B$ and $C$ are positive constants. In this case, we note that as

$$
\left|\frac{1}{2 \pi i} \int_{q-i \infty}^{q+i \infty} \Gamma(s) F(s ; 0) x^{-s} \mathrm{~d} s\right|=O\left(\left|\frac{x}{B}\right|^{m}\right) \quad\left(q=-m-\frac{1}{2}, m \rightarrow \infty\right),
$$

which shows that the IMT (3.9) is absolutely convergent when $0<x<B$. However, (3.9) remains well defined and uniformly convergent when $0<x_{0} \leq x \leq X<\infty$. Hence, 
the IMT (3.9) defines a regular function of $x(0<x<\infty)$ which also has the series representation

$$
F(0 ; x)=\sum_{n=0}^{\infty} \frac{(-1)^{n} F(-n ; 0) x^{n}}{n !} \quad(0<x<B) .
$$

In order to state Ramanujan's master theorem precisely and give the series representation (3.17) in the finite and infinite intervals of $x$, we need three classes of functions that are introduced in the next section.

\section{The classes of functions suitable for the master theorem}

We define three classes of functions suitable for the Hardy-Ramanujan formula. Our approach is different from Hardy's, as we define our classes by introducing an asymptotic relation satisfied by the product $\Gamma(s) F(s ; 0)$. This facilitates the determination of a particular function to which the Hardy-Ramanujan formula is applicable.

Definition A meromorphic function $F(s ; 0)$ is said to be a member of the class $Q_{\infty}(\varepsilon$, $\delta)(\varepsilon, \delta>0)$ if:
(a) $F(s ; 0)(\sigma<\infty)$ is analytic;
(b) $|\Gamma(s) F(s ; 0)| \leq C|\tau|^{\varepsilon(\sigma-\delta)}(\sigma<\delta)$.
(c) $F(s ; 0)$ is not identically zero at all the non-positive integers at $s=0,-1,-2,-3, \ldots$.

Definition A meromorphic function $F(s ; 0)$ is said to be a member of the class $Q_{P}(\varepsilon$, $\delta)(\varepsilon, \delta>0)$ if:
(a) $F(s ; 0)(\sigma<\delta)$ is analytic,
(b) $|\Gamma(s) F(s ; 0)| \leq C e^{-P \sigma-\varepsilon|\tau|}(\sigma<\delta)$.
(c) $F(s ; 0)$ is not identically zero at all the non-positive integers at $s=0,-1,-2,-3, \ldots$.

Definition A meromorphic function $F(s ; 0)$ is said to be a member of the class $R_{\infty}(\varepsilon$, $\delta)(\varepsilon, \delta>0)$ if:
(a) $F(s ; 0)(\sigma<\delta)$ is analytic,
(b) $|\Gamma(s) F(s ; 0)| \leq C|\tau|^{\sigma} e^{-\varepsilon|\tau|}(\sigma<\delta)$.
(c) $F(s ; 0)$ is not identically zero at all the non-positive integers at $s=0,-1,-2,-3, \ldots$.

Theorem (4.1) Every $F(s ; 0) \in Q_{\infty}(\varepsilon, \delta)$ (or $\left.F(s ; 0) \in R_{\infty}(\varepsilon, \delta)\right)$ satisfies (3.11), (3.12).

Theorem (4.2) Every $F(s ; 0) \in Q_{P}(\varepsilon, \delta)$ satisfies (3.17) for $B=\mathrm{e}^{p}$.

\section{Some applications of the Hardy-Ramanujan master theorem}

We now give examples that include functions that do not belong to Hardy's class for which Ramanujan's master theorem is applicable. As $\Gamma(s) \Gamma(1-s)=\pi / \sin (\pi s)$, using Hardy's notation we have

$$
\phi(-s)=F(s ; 0) / \Gamma(1-s) \text { and }(\pi / \sin (\pi s)) \phi(-s)=\Gamma(s) F(s ; 0),
$$

(see (1.6)). Therefore, it is natural to introduce classes of functions based on the asymptotic representations of the product $\Gamma(s) F(s ; 0)$ as we have done in Section 4 .

Example (5.1) Let

$$
\Gamma(s) F(s ; 0):=\Gamma(s) \zeta(s) \quad(0<\sigma<1) .
$$

Then, we note that $F(s, 0):=\zeta(s)(-\infty<\sigma<1)$ is analytic and for $0<\theta \leq \theta_{0}<\frac{\pi}{2}$ and (see [[9], pp. 135-136]) 


$$
|\Gamma(s) F(s, 0)| \leq C(2 \pi)^{\sigma} e^{-\left(\frac{\pi}{2}-\theta\right)|\tau|} \quad\left(|\tau| \rightarrow \infty,-\infty<\sigma_{1} \leq \sigma \leq \sigma_{2}<1\right) .
$$

Therefore, $F(s ; 0):=\zeta(s) \in Q_{\log (1 / 2 \pi)}\left(\frac{\pi}{2}-\theta_{0}, 1\right)$. Hence, according to the lemma (3.1), the IMT

$$
F(0 ; x)=\frac{1}{2 \pi i} \int_{c-i \infty}^{c+i \infty} \Gamma(s) \zeta(s) x^{-s} \mathrm{~d} s \quad(0<c<1)
$$

is well defined. The integrand in (5.3) has simple poles at $s=0,-1,-2, \ldots$. Using Cauchy's residue theorem, we get

$$
F(0 ; x)=\sum_{n=0}^{\infty} \frac{(-1)^{n} \zeta(-n) x^{n}}{n !} \quad(0<x<2 \pi),
$$

that can be simplified further as (see [[6], p. 264-266] and [[9], p. 136])

$$
F(0 ; x)=\frac{1}{\mathrm{e}^{x}-1}-\frac{1}{x}=\sum_{n=0}^{\infty} \frac{(-1)^{n} \zeta(-n) x^{n}}{n !} \quad(0<x<2 \pi) .
$$

Example (5.2) Let

$$
\Gamma(s) F(s ; 0):=\Gamma(s) \Gamma(1-s)=\frac{\pi}{\sin \pi s} \quad(0<\sigma<1) .
$$

Here the function $F(s ; 0)=\Gamma(1-s)(\sigma<1)$ is analytic and

$$
|\Gamma(s) F(s ; 0)|=|\Gamma(s) \Gamma(1-s)| \leq C e^{-\pi|\tau|} \quad\left(|\tau| \rightarrow \infty,-\infty<\sigma_{1} \leq \sigma \leq \sigma_{2}<1\right),
$$

which shows that $F(s ; 0):=\Gamma(1-s) \in Q_{0}(\pi, 1)$. Therefore, according to the lemma (3.1), the IMT

$$
F(0 ; x)=\frac{1}{2 \pi i} \int_{c-i \infty}^{c+i \infty} \Gamma(s) \Gamma(1-s) x^{-s} \mathrm{~d} s=\frac{1}{2 \pi i} \int_{c-i \infty}^{c+i \infty} \frac{\pi}{\sin \pi s} x^{-s} \mathrm{~d} s \quad\left(x>0,0<\sigma_{1} \leq c \leq \sigma_{2}<1\right),
$$

is well defined. Using the Cauchy's residue theorem we find

$$
F(0 ; x)=\frac{1}{1+x}=\sum_{n=0}^{\infty} \frac{(-1)^{n}}{n !} \Gamma(1+n) x^{n}=\sum_{n=0}^{\infty}(-1)^{n} x^{n} \quad(0<x<1),
$$

It is worth pointing out that Ramanujan's master theorem stated as

$$
\int_{0}^{\infty} x^{s-1}\left(\sum_{n=0}^{\infty} \frac{(-1)^{n} \Gamma(1+n)}{n !} x^{n}\right) \mathrm{d} x=\Gamma(s) \Gamma(1-s)=\frac{\pi}{\sin \pi s} \quad(0<\sigma<1)
$$

is not meaningful, as the series in (5.9) and (5.10) is divergent if $x>1$. However, the IMT (5.8) is well defined that has the series representation (5.9). The series representation in (5.10) must be replaced by the analytic continuation on the LHS of (5.9).

Example (5.3) We now give an example of a function where Ramanujan's master theorem is applicable but the function does not belong to Hardy's class of functions. 
Consider $F(s ; 0):=\sin \left(\frac{\pi s}{2}\right)$. Then we note that

$$
\left|\Gamma(s) \sin \left(\frac{\pi s}{2}\right)\right|=O\left(|\tau|^{\sigma-\frac{1}{2}}\right) \quad\left(|\tau| \rightarrow \infty,-\infty<\sigma_{1} \leq \sigma \leq \sigma_{2}<1 / 2\right) .
$$

Hence, according to the above lemma (3.1), the line integral

$$
F(0 ; x):=\frac{1}{2 \pi i} \int_{c-i \infty}^{c+i \infty} \Gamma(s) \sin \left(\frac{\pi s}{2}\right) x^{-s} \mathrm{~d} s \quad\left(x>0,0<c_{1} \leq c \leq c_{2}<1 / 2\right),
$$

is well defined. From Cauchy's residue theorem, the integral (5.11) can be evaluated by taking the sum of the residues at the simple poles at $s=-1,-3,-5, \ldots$ leading to a closed form $f(x)=\sin x$. Hence, we have the Mellin transform representation

$$
M[\sin x ; s]:=\int_{0}^{\infty} \frac{\sin x}{x^{1-s}} \mathrm{~d} x=\Gamma(s) \sin \left(\frac{\pi s}{2}\right) \quad(0<\sigma<1 / 2) .
$$

We note that $F(s ; 0) \in Q_{\infty}(1,1 / 2)$. Following Jordan's lemma, the integral (5.13) remains convergent for $0<\sigma<1$ which shows that (3.9) is valid in the strip $0<\sigma<1$. Note that in this example we have $A=\pi$ and $\phi(-s)=F(s ; 0) / \Gamma(1-s)=\sin \left(\frac{\pi s}{2}\right) / \Gamma(1-s)$ which shows that $\varphi$ does not belong to Hardy's class of functions.

Example (5.4) We give a second example of a function where Ramanujan's master theorem is applicable but the function does not belong to Hardy's class of functions. The applicability of the Ramanujan's master theorem is assured by the lemma (3.1). Consider $F(s ; 0)=\frac{1}{\Gamma(1-s)}(\sigma<1 / 2)$. Then (see [[7], p. 6(1.45)])

$$
\left|\frac{\Gamma(s)}{\Gamma(1-s)}\right|=O\left(|\tau|^{2\left(\sigma-\frac{1}{2}\right)}\right) \quad\left(|\tau| \rightarrow \infty,-\infty<\sigma_{1} \leq \sigma \leq \sigma_{2}<1 / 2\right) .
$$

Hence, according to the above lemma, the integral

$$
\begin{aligned}
& F(0 ; x):=\frac{1}{2 \pi i} \int_{c-i \infty}^{c+i \infty} \frac{\Gamma(s)}{\Gamma(1-s)} x^{-s} \mathrm{~d} s \\
& \left(x>0,0<c_{1} \leq c \leq c_{2}<1 / 2\right),
\end{aligned}
$$

is well defined. From Cauchy's residue theorem, the integral (5.15) can be evaluated by taking the sum of the residues at the simple poles at $s=0,-1,-2, \ldots$ leading to

$$
F(0 ; x)=\sum_{n=0}^{\infty} \frac{(-1)^{n} x^{n}}{(n !)^{2}}
$$

which has the closed form $F(0 ; x)=J_{0}(2 \sqrt{x})$. Again we have $A=\pi$ and

$$
\phi(-s)=F(s ; 0) / \Gamma(1-s)=1 /(\Gamma(1-s))^{2},
$$


which shows that $\varphi$ does not belong to Hardy's class of functions. However, $F(\mathrm{~s} ; 0)$ $\in Q_{\infty}(2,1 / 2)$.

Example (5.5) We give another example of a function involving ratio of the Riemann zeta functions where our lemma (3.1) assures the application of Ramanujan's master theorem but the function does not belong to Hardy's class of functions. Consider

$$
\begin{aligned}
F(s ; 0) & :=\frac{\zeta(s)}{\Gamma(1-s) \zeta(1-s)}(\sigma<1 / 2) \text {. Then (see [[5], p. 81]) } \\
& \left|\frac{\Gamma(s) \zeta(s)}{\Gamma(1-s) \zeta(1-s)}\right|=O\left(|\tau|^{\sigma-\frac{1}{2}}\right) \quad\left(|\tau| \rightarrow \infty,-\infty<\sigma_{1} \leq \sigma \leq \sigma_{2}<1 / 2\right) .
\end{aligned}
$$

Hence, according to the above lemma, the integral

$$
F(0 ; x):=\frac{1}{2 \pi i} \int_{c-i \infty}^{c+i \infty} \frac{\Gamma(s) \zeta(s)}{\Gamma(1-s) \zeta(1-s)} x^{-s} \mathrm{~d} s \quad\left(x>0,0<c_{1} \leq c \leq c_{2}<1 / 2\right)(5.1
$$

is well defined. From Cauchy's residue theorem, the integral (5.16) can be evaluated by taking the sum of the residues at the simple poles at $s=-1,-3, \ldots$ leading to

$$
F(0 ; x)=\sum_{n=0}^{\infty} \frac{(-1)^{n} \zeta(-n) x^{n}}{(n !)^{2} \zeta(1+n)} .
$$

The series (5.19) can be simplifies further by using Riemann functional equation (1.11) to give the closed form

$$
F(0 ; x)=\sum_{n=0}^{\infty} \frac{(-1)^{n} \zeta(-n) x^{n}}{(n !)^{2} \zeta(1+n)}=\frac{1}{\pi} \sin \left(\frac{\pi x}{2}\right) .
$$

Once again we have

$$
\phi(-s)=F(s ; 0) / \Gamma(1-s)=\zeta(s) / \zeta(1-s)(\Gamma(1-s))^{2},
$$

which shows that $\varphi$ does not belong to Hardy's class of functions. However, $F(\mathrm{~s} ; 0)$ $\in Q_{\infty}(1,1 / 2)$.

Example (5.6) Consider

$$
F(s ; 0):=\frac{\zeta(1-s)}{\Gamma(s) \zeta(s)} \quad(\sigma<1 / 2)
$$

Then we note that (see [[5], p.81])

$$
|\Gamma(s) F(s ; 0)|=\left|\frac{\zeta(s)}{\zeta(1-s)}\right|=|\chi(s)|=O\left(|\tau|^{\sigma-\frac{1}{2}}\right) \quad\left(|\tau| \rightarrow \infty,-\infty<\sigma_{1} \leq \sigma \leq \sigma_{2}<1 / 2\right) .
$$

Hence, according to the lemma (3.1), the IMT

$$
F(0 ; x)=\frac{1}{2 \pi i} \int_{c-i \infty}^{c+i \infty} \frac{\zeta(1-s)}{\zeta(s)} x^{-s} \mathrm{~d} s \quad(0<c<1 / 2, x \geq 0)
$$

is well defined. The integral (5.23) can be evaluated by Cauchy's residue theorem which has the closed form (see [[13], p. 91(3.3.6)]), $F(0 ; x)=2 \cos (\pi x)$. Once again the function 


$$
\phi(-s)=F(s ; 0) / \Gamma(1-s)=\zeta(1-s) / \zeta(s) \Gamma(s) \Gamma(1-s),
$$

which shows that $\varphi$ does not belong to Hardy's class of functions. However, $F(\mathrm{~s} ; 0)$ $\in Q_{\infty}(1,1 / 2)$.

\section{Different forms of the Hardy-Ramanujan master theorem}

The Hardy-Ramanujan master theorem can be represented in different forms by considering special cases of the function $F(s ; 0)$ (see [[2], p. 186]) in the above lemma and in theorems (4.1) and (4.2). These special cases were stated as formulas/theorems by Ramanujan. These are special cases of the master theorem. We state them in the form of theorems for the sake of completeness.

Theorem (6.1) Let

$$
F(s ; 0):=\Gamma(1-s) G(s),
$$

be in one of the above three classes. Then, we have

$$
\begin{aligned}
& \frac{1}{2 \pi i} \int_{c-i \infty}^{c+i \infty} \Gamma(s) \Gamma(1-s) G(s) x^{-s} \mathrm{~d} s=\frac{1}{2 \pi i} \int_{c-i \infty}^{c+i \infty} \frac{\pi}{\sin \pi s} G(s) x^{-s} \mathrm{~d} s \quad(0<c<1) . \\
& =\sum_{n=0}^{\infty}(-1)^{n} G(-n) x^{n}
\end{aligned}
$$

Theorem (6.2) Let

$$
F(s ; 0):=\Gamma(1-s) G(s) H(1-s)
$$

be in one of the above three classes. Then, we have

$$
\begin{aligned}
& \frac{1}{2 \pi i} \int_{c-i \infty}^{c+i \infty} \Gamma(s) \Gamma(1-s) G(s) H(1-s) x^{-s} \mathrm{~d} s=\frac{1}{2 \pi i} \int_{c-i \infty}^{c+i \infty} \frac{\pi}{\sin \pi s} G(s) H(1-s) x^{-s} \mathrm{~d} s \\
& =\sum_{n=0}^{\infty}(-1)^{n} G(-n) H(1+n) x^{n} \\
& \quad(0<c<1) .
\end{aligned}
$$

Theorem (6.3) (Extension of Ramanujan's Formulas)

Let $F(s ; 0) G(1-s ; 0)$ be in one of the three classes. Then

$$
\begin{aligned}
& F(0 ; x) \circ G(0 ; x):=\int_{0}^{\infty} F(0, x t) G(0, t) \mathrm{d} t=\frac{1}{2 \pi i} \int_{c-i \infty}^{c+i \infty} \frac{\pi}{\sin \pi s} F(s ; 0) G(1-s ; 0) x^{-s} \mathrm{~d} s \\
& =\sum_{n=0}^{\infty}(-1)^{n} F(-n ; 0) G(1+n ; 0) x^{n} .
\end{aligned}
$$

Proof Since we have

$$
M[f(x) \circ g(x) ; s]=f_{M}(s) g_{M}(1-s),
$$

which implies that

$$
\begin{aligned}
& M[F(0 ; x) \circ G(0 ; x) ; s] \\
& =\Gamma(s) \Gamma(1-s) F(s ; 0) G(1-s ; 0)=\frac{\pi}{\sin \pi s} F(s ; 0) G(1-s ; 0) .
\end{aligned}
$$


Taking the IMT in (6.6), we arrive at (6.5).

Remark (6.4) It may be noted that the result (6.4) can be regarded as an extension of Ramanujan's Formulas (D.1) and (D.2) (see [[2], p. 186]). Putting $F(0 ; x)=\cos x$ and $F(s ; 0)=\cos \left(\frac{\pi s}{2}\right)$ in (6.6), we obtain

$$
\begin{aligned}
& \cos (x) \circ G(0 ; x):=\int_{0}^{\infty} \cos (x t) G(0 ; t) \mathrm{d} t=\frac{1}{2 \pi i} \int_{c-i \infty}^{c+i \infty} \frac{\pi}{\sin \pi s} \cos \frac{\pi s}{2} G(1-s ; 0) t^{-s} \mathrm{~d} s \\
& =\sum_{n=0}^{\infty}(-1)^{n} G(1+2 n ; 0) x^{2 n},
\end{aligned}
$$

which is (D.1) (see [[2], p. 187]).

Putting $F(0 ; x)=\sin x$ and $F(s ; 0)=\sin \left(\frac{\pi s}{2}\right)$ in (6.6), we obtain

$$
\begin{aligned}
& \sin (x) \circ G(0 ; x):=\int_{0}^{\infty} \sin (x t) G(0 ; t) \mathrm{d} t=\frac{1}{2 \pi i} \int_{c-i \infty}^{c+i \infty} \frac{\pi}{\sin \pi s} \sin \frac{\pi s}{2} G(1-s ; 0) x^{-s} \mathrm{~d} s \\
& =\sum_{n=0}^{\infty}(-1)^{n} G(2+2 n ; 0) x^{2 n+1},
\end{aligned}
$$

which is (D.2).

\section{Concluding remarks}

Rigorous proofs are needed not only to be absolutely sure of the validity of the result but also to clearly define the range of its validity. This is, of course, necessary for actual application of the result. Even more importantly, the clear statement of the limitations delineates the precise reason for those limitations and hence provides the direction to be taken to proceed beyond those limits. Ways may then be found of 'bypassing' those limitations or of going around the limitations by extending the domain of the variables and thereby entering a different domain from the original, as done in analytic continuation. This has been the philosophy behind the present work.

In this article, we have provided a rigorous proof of the Hardy-Ramanujan theorem for a wider class of functions than was previously available. In fact, Hardy had noted that the theorem should apply to a wider class of functions but had then provided the extension. He does not appear to have provided the required extension later either. It may be thought that this indicates that he felt that the extension was not worth pursuing but, as we have pointed out here, many functions that are likely to be used in the application of the master theorem and Ramanujan's formula lie outside the domain of classes defined by Hardy. As such, the need for that extension was there but had not been provided. As we show, the rigorous proof for the wider class leads to even more general applicability of the theorem.

It would be worth seeing if the theorem could be extended still further. This extension is being explored separately. It appears that an extension may be possible. 
Acknowledgements

The authors are grateful to the King Fahd University of Petroleum and Minerals for providing excellent research facilities through IN090049.

\section{Author details}

'Department of Mathematics and Statistics, King Fahd University of Petroleum and Minerals, Dhahran, 31261, Saudi Arabia ${ }^{2}$ Center for Advanced Mathematics and Physics, National University of Science and Technology, H-12,

Islamabad, Pakistan

\section{Authors' contributions}

Both authors take full joint responsibility for the paper. There are equal contributions of both authors.

\section{Competing interests}

The authors declare that they have no competing interests.

Received: 2 December 2010 Accepted: 1 March 2012 Published: 1 March 2012

\section{References}

1. Berndt, BC: Ramanujan's Notebooks, Part IV. Springer-Verlag, New York. (1989)

2. Hardy, GH: Ramanujan: Twelve Lectures on Subjects Suggested by His Life and Work. Chelsea Publishing Company, New York. (1959)

3. Edwards, HM: Riemann Zeta Function. Academic Press, New York (1974)

4. Ash, RB: Complex Variables. Academic Press New York and London (1971)

5. Titchmarsh, EC: The Theory of the Riemann Zeta Function. Oxford University Press, New York (1951)

6. Apostol, TM: Introduction to Analytic Number Theory. Springer-Verlag, New York Heidelberg Berlin. (1976)

7. Chaudhry, MA, Zubair, SM: On a Class of Incomplete Gamma Functions with Applications. Chapman and Hall/CRC. (2001)

8. Davenport, H: Multiplicative Number Theory, second edition. New York, Springer-Verlag. (1980)

9. Hardy, GH, Littlewood, JE: Contributions to the Theory of the Riemann Zeta Function and the Theory of the Distribution of Primes. Acta Math. 41, 119-196 (1918)

10. Magnus, W, Oberhettinger, F, Soni, RP: Formulas and Theorems for the Special Functions of Mathematical Physics. Springer-Verlag, New York. (1966)

11. Srivastava, HM: Some formulas for the Bernoulli and Euler polynomials at rational arguments. Math Proc Camb Philos Soc. 129, 77-84 (2000). doi:10.1017/S0305004100004412

12. Temme, NM: Special Functions: An Introduction to Classical Functions of Mathematical Physics. Wiley, New York. (1996)

13. Paris, RB, Kaminski, D: Asymptotics and Mellin-Barnes Integrals. Cambridge University Press, Cambridge (2001)

14. Miller, KS, Ross, B: An Introduction to the Fractional Calculus and Fractional Differential Equations. John Wiley \& Sons, Inc., New York (1993)

doi:10.1186/1029-242X-2012-52

Cite this article as: Chaudhry and Qadir: Extension of Hardy's class for Ramanujan's interpolation formula and master theorem with applications. Journal of Inequalities and Applications 2012 2012:52.

\section{Submit your manuscript to a SpringerOpen ${ }^{\odot}$ journal and benefit from:}

Convenient online submission

- Rigorous peer review

- Immediate publication on acceptance

- Open access: articles freely available online

- High visibility within the field

- Retaining the copyright to your article

Submit your next manuscript at $\gg$ springeropen.com 